world, and was a director in the Parisian Gas Company and the Eastern Railway of France. His family relations were singularly happy. He leaves behind him a group of five sons. In addition to the treatise on aluminium already alluded to, Deville was the author, in company with Débray, of an exhaustive work in two volumes on the "Métallurgie du Platine" (1863).

T. H. N.

\section{CONVERSAZIONE AT KING'S COLLEGE}

$\mathrm{N}$ Saturday, July 2 , a brilliant and successful conversazione, given by the Council and the Academical Staff of King's College, brought to a conclusion the celebration of the fiftieth anniversary of the opening of the College. In the afternoon H.R.H. the Prince of Wales, accompanied by H.R.H. the Princess of Wales, distributed the College prizes to successful students, and the College rooms were converted into tastefully decorated drawing-rooms and picture galleries, in which were exhibited many very choice pictures and works of art.

The library was furnished with microscopes which had been lent by members of the Microscopic Society. The large entrance hall and the front of the College were brilliantly lighted by three Crompton electric lights, which burnt with remarkable steadiness throughout the evening. In the scientific department, the museum of King George III. contains an unrivalled collection of mechanical and physical apparatus, and is especially rich in apparatus of historic interest. The nucleus of the collection was presented to the College by Her Majesty the Queen in I 843, when the museum was opened by Prince Albert, who then witnessed some of the experiments of Sir Charles Wheatstone on the electric telegraph. Important additions have been made to the collection of apparatus by the Professors of Natural Philosophy, and at his death Sir Charles Wheatstone's valuable collection was bequeathed to the museum. Among the interesting features in the museum are: calculating machines of Cavendish and others, Appoldie centrifugal pump, Newcomen's model of his steam-engine, original forms of Daniell's battery, Siberian loadstone used for his induction spark by Faraday, original Wheatstone's bridge, early forms of stereoscope, early forms of electrical machines, polar clocks and shadow clocks, De Kempelen's talking machine.

From its foundation in 1868 the Physical Laboratory, now called the Wheatstone Laboratory, has been under the direction of Prof. W. Grylls Adams. Among the interesting apparatus exhibited in this department were the Wheatstone Collection of electrical apparatus for exhibition in Paris, dynamo-electric machines, diffraction spectra, an optical bench, showing interference of light, measuring polariscopes, with universal motions for the exact measurement of crystals, and vacuum tubes in great variety, including a very beautiful coronet. The great event of the evening in the Physical Department was the exhibition for the first time in England of the Faure's secondary battery or reservoir of electricity. Two boxes of this battery, which had been previously charged from a dynamo-electric machine, and had then been brought to the College, were capable of heating and keeping heated to bright redness a platinum wire 2 metres long and I millimetre in diameter. Six boxes were found to be sufficient to cause Swan electric lamps to glow brilliantly. Twelve of these boxes supplied a pedestal of Lane-Fox lamps, supplied by the British Electric Light Company, and during the evening the Physical Lecture Theatre was brilliantly illuminated by twenty Swan lamps of the latest type with the current from twelve other boxes of Faure's secondary battery. It was shown that by means of these boxes of electricity the lighting of private houses by electricity was already an accomplished fact.
THE COMET

$W^{E}$ have received the following communications :-

AT about IIb. Om. G.M.T. on June 29 a transit of the "following" nuclear jet of the great comet over a star of $8 \mathrm{~m}$. was observed by Mr. N. F. Green, of 39, Circus Road, St. John's Wood, and by me, with a $12 \frac{1}{2}$-inch reflector belonging to Mr. Green. Definition was very good and tranquil. As the star became involved in the jet it gradually increased in size, and when seen through the brightest part of the jet traversed resembled an illdefined planetary disk about $3^{\prime \prime}$ in diameter. At this moment the comet seemed to have two nuclei similar in aspect and brightness.

The effect of the cometary matter on the star's image resembled that of ground glass, not that of fog; the image of the star, being dilated into a patch of nearly uniform brightness, instead of presenting a sharp central point with a surrounding halo. Cirro-stratus, passing into raincloud,'produces on the appearance of the sun an effect the counterpart of that produced by the cometary emitted matter on the star. There was not sufficient light for the use of the spectroscope, the star, afterwards identified as B.D. $+65^{\circ}, 519$, being fainter than $8 \mathrm{~m}$.

The transit of the jet occupied about $3 \mathrm{~m}$., and the star slowly resumed its ordinary appearance and dimensions, the image contracting as the centre of the jet left the star behind. A transit of this kind has not frequently been observed, at least under such favourable conditions as to brightness and definition of the objects, and it is to be hoped that others may have been as fortunate as Mr. Green and the undersigned.

If the point which obeys the Newtonian law be a solid body, the observation just recorded seems to show that its true outline would probably be rendered unrecognisable, and its aspect totally altered by the (refractive?) power of the coma and jets.

38, Barclay Road, S.W., July I

THE following is an extended list of places obtained with the transit-circle when the comet passed sub Polo:-

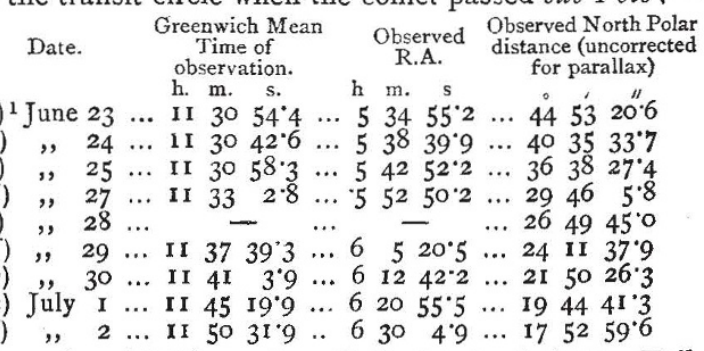

Remarks. - (a) The nucleus distinct but nebulous. Tail bright, and estimated $15^{\circ}$ in length. Observation good.

(b) Observation difficult, owing to cloud.

(c) Nucleus better defined than on June 23, but not so bright. Length of tail estimated at $15^{\circ}$. Observation good.

(d) Observation fair, very cloudy. Tail $12^{\circ}-15^{\circ}$ long.

(e) Observed through short break in clouds. Tail $10^{\circ}$ in length.

(f) Observation very good. Tail $10^{\circ}$.

(g) Observation very good. Nucleus smaller and fainter than on preceding nights. Tail $10^{\circ}$.

(h) Observation very good. Tail $9^{\circ}$.

(i) Very faint, observed through haze. Tail $8^{\circ}$.

Radcliffe Observatory, Oxford

E. J. STONE

MY chief object in writing to-day is to explain a word in my letter of June 28 (p. 200) that is quite open to misinterpretation. In examining the head of Comet $b$ I88 I with a small direct-vision spectroscope and a narrow slit, I saw, on June 27 , three bright lines or bands on a faint continuous spectrum. Two of the lines were strong and

I The observed R.A. and G.M.T. for June 23, reported in last week's NATURE (p. 200), should be decreased one minute, as above. 